\title{
Quantitative assessment of airway and parenchymal components of chronic obstructive pulmonary disease using thin-section helical computed tomography
}

\author{
Narvir S. Chauhan ${ }^{A, B, C, D, E, F}$, Dinesh Sood ${ }^{A, E, F}$, Preeti Takkar ${ }^{A, B, C, D, E, F}$, Devendra S. Dhadwal ${ }^{A, D, E}$, Rajiv Kapila ${ }^{A, B, E, F}$ \\ Dr Rajendra Prasad Government Medical College Tanda, India
}

\section{Abstract}

Purpose: The purpose of this study was to diagnose and characterise chronic obstructive pulmonary disease (COPD) into its forms, patterns, and severity using MDCT.

\begin{abstract}
Material and methods: In this prospective study, spirometric and MDCT evaluation was done in 52 consecutive patients diagnosed with COPD. In each patient six segmental bronchi were evaluated for CT morphometric indices of bronchial wall thickness (BWT) and wall area percentage (WAP). Quantitative evaluation of emphysema was done using inbuilt software, and volume of emphysematous lung was determined using percentage low attenuation area (LAA). COPD was categorised into the following: emphysema predominant; airway predominant; or mixed phenotypes, and severity grading was assigned as mild, moderate, or severe.

Results: Centrilobular was the predominant emphysema pattern occurring alone (36.5\%) or in combination with paraseptal changes (34.6\%). Among COPD phenotypes, emphysema predominant was the commonest (44.3\%), followed by mixed (30.8\%), and bronchitis predominant (25.0\%). The mean BWT in the airway-predominant group was significantly higher $(1.94 \pm 0.28 \mathrm{~mm})$ than in the emphysema-predominant subgroup $(1.79 \pm 0.23 \mathrm{~mm})$ with a $p$ value of 0.005 .
\end{abstract}

Conclusion: MDCT is an indispensable tool in quantitative and qualitative evaluation of COPD patients. Measurement of CT indices like BWT, WAP, and \%LAA can reliably categorise COPD into phenotypes like emphysema predominant, airway predominant, or mixed, which serve as a guide for patient management.

Key words: COPD, pulmonary emphysema, chronic bronchitis, spirometry.

\section{Introduction}

Chronic obstructive pulmonary disease (COPD) is a significant contributor to morbidity and mortality worldwide. It is estimated that COPD will become the fourth leading cause of death by the year 2030 [1]. COPD is typified by the occurrence of an airway obstruction, which is non-reversible on bronchodilator administration and occurs secondarily to airway narrowing, emphysema, or a combination of both [2]. Previous studies have focused either on quantification of pulmonary emphysema or on airway wall assessment using CT-based indices [3,4]. In the actual scenario, however, the majority of cases encountered in clinical practice have varying components of both phenotypes. This study was done with the aim of prospectively quantifying both the components in patients of COPD in the North Indian population subgroup.

\section{Material and methods}

This is a prospective study conducted during a span of one year in our institution, after approval from the in-

Correspondence address:

Dr. Preeti Takkar, Dr Rajendra Prasad Government Medical College Tanda, India, phone: +919418476622, e-mail: drpreetirpgmc@gmail.com

Authors' contribution:

A Study design · B Data collection · C Statistical analysis · D Data interpretation · E Manuscript preparation · F Literature search · G Funds collection 
stitutional Ethical Committee, and after informed consent was gained from patients for their voluntary participation. A total of 52 consecutive patients diagnosed clinically with COPD using American Thoracic Society criteria and fulfilling the inclusion and exclusion criteria were included in the study. The patients were first examined clinically in the Department of Pulmonary Medicine and diagnosed as COPD. Following this, spirometry and MDCT imaging of the chest was done on the same day. Prior to CT examination, data were collected with regard to anthropometric parameters, smoking index, and body mass index (BMI).

\section{Imaging procedure}

Helical non-contrast CT scan was performed on a 16-slice MDCT scanner (Philips Brilliance) in suspended full inspiration using the following parameters: $\mathrm{kVp}-120$; mAs - 200; slice thickness $-3 \mathrm{~mm}$; gantry tilt -0 degrees; Matrix $-512 \times 512$. Post processing was done using the inbuilt software application "Lung Emphysema". A colour-coded display of emphysematous areas in both lungs was obtained, and calculation results table and histograms were generated. The percentage LAA obtained was used to determine the grade of emphysema severity, and patients were grouped as mild (0-5\%), moderate (5-30\%), and severe $(>30 \%)$.

Multiplanar reconstruction was done to align the image of the airway along its true longitudinal axis and one bronchial branch from each lobe (RUL, RML, RLL, LUL, lingular segments, and LLL) was selected for assessment in the true transverse plane. Various airway dimensions were then measured separately by two radiologists with twenty and nine years of experience in chest CT imaging, and the averages of the readings were given. A minimum of six segmental bronchi having external diameters more than $2 \mathrm{~mm}$ were evaluated manually on Lung Window using the previously-described CT indices [4]. The outer wall diameter (D) and inner wall/luminal diameter (L) were determined, and then the BWT and PWA were calculated

Table 1. Anthropometric and spirometric data distribution in the study population

\begin{tabular}{|l|c|c|}
\hline Parameter & Mean \pm SD & Range \\
\hline Age (years) & $64.17 \pm 10.51$ & $35-83$ \\
\hline Height $(\mathrm{cm})$ & $160.15 \pm 7.74$ & $146-177$ \\
\hline Weight $(\mathrm{kg})$ & $47.26 \pm 8.36$ & $33-78$ \\
\hline BMl & $18.42 \pm 2.95$ & $13.05-27.96$ \\
\hline Smoking Index (Jindal) & $562.89 \pm 369.66$ & $50-1440^{*}$ \\
\hline FEV1 (\% predicted) & $40.55 \pm 19.24$ & $12-88$ \\
\hline FVC (\% predicted) & $59.94 \pm 22.21$ & $23-133$ \\
\hline FEV1/FVC (\%) & $64.61 \pm 13.39$ & $34-95$ \\
\hline
\end{tabular}

*Five non-smoking females with Biogas smoke exposure were not included using the following formulas: $\mathrm{PWA}=\pi(\mathrm{D} / 2)^{2}-\pi(\mathrm{L} / 2)^{2} /$ $\pi(\mathrm{D} / 2)^{2} \times 100 ; \pi(D / 2)^{2}$ for the total area, where $\pi(L / 2)^{2}$ is the endoluminal area. BWT was used to grade the severity of the airway disease into mild (1-2 mm), moderate (2-3 $\mathrm{mm})$, and severe (>3 mm).

\section{Statistical analysis}

Statistical analysis was done using IBM SPSS software (Version 23). The continuous variables were expressed as mean \pm SD. The Mann-Whitney test was used to compare the airway measurements, MAA values, and PFT results between the various disease groups. Results were considered significant at $p$ value $<0.05$

\section{Ethical statement}

This study was approved by the institutional Ethics Committee. All procedures were performed in accordance with the ethical standards of our institute and also in accordance with the 1964 Helsinki Declaration and its later amendments.

\section{Informed consent}

Informed consent was obtained from all participants for their voluntary participation in the study.

\section{Results}

The mean age of patients in our study was 64 years with maximum number of patients in the age group of 61-70 years. Out of 52 patients, there were 47 males and five female patients with a male: female ratio of 9.3:1. The mean $\mathrm{BMI}$ in the study was $18.42(\mathrm{SD} \pm 2.95)$ with a range of 13.04-27.0. All male subjects were smokers, and all female patients had a history of exposure to biomass fuel. The smoking index, calculated for males according to formula used by Jindal et al. [5] (SI = No. of cigarettes/bidis per day $\times$ total duration), showed mean SI of 562.89 S.D \pm 369.66 with the maximum number of patients $(61.53 \%)$ having SI > 300 (Table 1). Comparison between BMI and SI values yielded a $p$ value of 0.01 , establishing a statistically significant correlation.

Spirometric examination yielded a mean FEV1 (\% predicted) of $40.55 \mathrm{SD} \pm 19.24$, mean FVC (\% predicted) of $59.94 \mathrm{SD} \pm 22.21$, and mean FEV1/FVC ratio of 64.61 $\pm 13.39 \%$ (Table 1 ). Correlation of FEV1/FVC ratio with CT severity grading of emphysema was statistically significant, with a $p$ value of 0.034 . A similar correlation between FEV1/FVC and BWT was, however, not observed.

The emphysema software post processed data yielded a mean \%LAA of $11.78 \pm$ SD 11.81. There was also more severe involvement of the left lung, which showed a mean emphysematous volume of $294.63 \mathrm{cc}$ as compared to a volume of $228.11 \mathrm{cc}$ in the right lung (Table 2). 
In our study, the airway CT morphometric parameters of BWT and PWA were found to be increased (overall mean of $1.89 \mathrm{SD} \pm 0.42 \mathrm{~mm}$ for BWT and $80.83 \mathrm{SD}$ $\pm 6.22 \%$ for PWA) (Table 3). BWT was observed to be higher in the airway-predominant group (mean thickness $1.94 \pm 0.28 \mathrm{~mm}$ ) and lower in the emphysema-predominant subgroup (mean thickness of $1.79 \pm 0.23 \mathrm{~mm}$ ). The difference in two groups was statistically significant with $p$ value of 0.005 .

Using the obtained CT based parameters of \%LAA and BWT; we subcategorised our patients into emphysema-predominant, bronchitis-predominant, and mixed phenotypes, depending on the severity of their predominant findings (Table 4 ). The maximum number of patients $(N=16)$ were seen in the subcategory of emphysema predominant moderate, followed by mixed mild $(N=14)$, mixed moderate $(N=8)$, and emphysema predominant severe $(N=7)$.

\section{Discussion}

Chronic obstructive pulmonary disease is an obstructive disorder of peripheral airways, which occurs as a result of inflammatory response that causes remodelling of small airways or destruction of lung parenchyma.

Smoking is a common risk factor, which incites these slowly progressive inflammatory changes. In our study, which included 52 consecutive patients of clinically diagnosed COPD; all males patients $(N=47)$ had a history of smoking $(N=47)$, while all female patients had a history of biofuel gas exposure $(N=5)$. Mean smoking index for male patients was $562.89 \pm 369.66$. Expectedly, the patients with high SI values had frail build with lower BMI values especially in patients with emphysema-predominant phenotype, and the correlation between the two variables was found to be statistically significant. This observation is in corroboration with previous studies, which showed similar correlation between BMI and emphysema [6,7].

In addition to smoking, advancing age has also been considered as a significant factor in the prediction of clinically significant COPD. This was also corroborated in our study because the majority of patients were elderly, falling in age range of 61-70 years, and with a mean age of 64 years $(S D \pm 10.51)$.

Gender-specific differences in clinical expression of COPD are known, and females have been considered more likely to develop airway-predominant disease in compar-
Table 2. Distribution and quantification of emphysema

\begin{tabular}{|l|c|c|}
\hline Parameter & Mean \pm SD & Range \\
\hline Total lung volume & $4093.88 \pm 903.88$ & $1968-4217$ \\
\hline Right lung volume & $2153 \pm 472.56$ & $967-3406$ \\
\hline Left Lung volume & $1939.63 \pm 516.81$ & $963-3090$ \\
\hline Total emphysematous lung volume & $526.22 \pm 578.94$ & $1.10-1893$ \\
\hline Right lung emphysematous volume & $228.11 \pm 247.63$ & $1.0-926$ \\
\hline Left lung emphysematous volume & $294.63 \pm 357.52$ & $1.0-1363$ \\
\hline LAA\% & $11.78 \pm 11.81$ & $1.0-38$ \\
\hline
\end{tabular}

ison to males, who commonly have emphysema-predominant subtype [8]. Gender-based variations were also witnessed in our study, and the male patients (constituting $90.38 \%$ of the study group) showed an emphysema pattern in the majority $(N=22 ; 46.80 \%)$, followed by mixed $(N=23 ; 27.65 \%)$ and bronchitis patterns $(N=12 ; 25.53 \%)$. Females constituted only a small number of patients in the study group (9.62\%), and interestingly the majority of them showed a mixed pattern $(60.0 \%)$ instead of the expected airway pattern. The reason for this variation could be a history of exposure to biomass fuel smoke in the female patients in our study, which led to the coexistence of emphysema with airway disease.

Spirometry has traditionally been used to diagnose the COPD, and GOLD has defined COPD as FEV1/VC $<0.7$ after bronchodilator administration. Its utility is, however, limited because it provides functional information only and cannot reliably distinguish the airway-predominant COPD from the emphysema-predominant subtypes.

With the advent of newer-generation multislice CT, it has become possible to categorise COPD into its subtypes, as done in previous studies [9-11]. In our study helical CT was carried out on the same day as the spirometric examination using standard parameters and protocols, and all the COPD patients were subcategorised into various phenotypes, and their further grading based on severity of findings was done.

In patients with emphysema, CT provides information on the various subcategories of emphysema and their regional distribution. Quantification of emphysema can be done using threshold techniques expressed as low attenuation area (LAA\%). Automatic segmentation using software tools can then express the results on lobar basis. We used the inbuilt emphysema software tool with the result expressed as the percentage of LAA, for emphysema

Table 3. Morphometric indices of airway disease

\begin{tabular}{|c|c|c|c|c|c|c|c|}
\hline \multirow[t]{2}{*}{ CT indices } & \multicolumn{6}{|c|}{ Level of segmental bronchi } & \multirow[t]{2}{*}{ Overall mean } \\
\hline & RUL & RML & RLL & LUL & Lingular & LLL & \\
\hline $\operatorname{TDR}(\mathrm{cm})$, mean \pm SD & $0.32 \pm 0.07$ & $0.34 \pm 0.05$ & $0.34 \pm 0.06$ & $0.32 \pm 0.07$ & $0.33 \pm 0.05$ & $0.33 \pm 0.05$ & $0.33 \pm 0.05$ \\
\hline PWA (\%), \pm SD & $81.42 \pm 9.11$ & $83.10 \pm 7.91$ & $81.26 \pm 7.73$ & $78.95 \pm 10.07$ & $82.32 \pm 8.11$ & $81.30 \pm 8.26$ & $81.39 \pm 8.53$ \\
\hline BWT (mm), \pm SD & $1.83 \pm 0.33$ & $1.84 \pm 0.46$ & $2.05 \pm 0.52$ & $1.85 \pm 0.36$ & $1.87 \pm 0.42$ & $1.93 \pm 0.47$ & $1.89 \pm 0.42$ \\
\hline
\end{tabular}


Table 4. Classification of COPD into subcategories

\begin{tabular}{|c|c|c|c|}
\hline \multirow[b]{2}{*}{$\begin{array}{l}\text { Bronchial wall } \\
\text { thickness (B) }\end{array}$} & \multicolumn{3}{|c|}{ Emphysema (E) } \\
\hline & Mild (m) & Moderate (M) & Severe (S) \\
\hline Mild (m) & $\begin{array}{c}\mathrm{mEmB} \\
\text { Mixed mild } \\
14\end{array}$ & $\begin{array}{c}\text { MEmB } \\
\text { Epred moderate } \\
16\end{array}$ & $\begin{array}{c}\text { SEmB } \\
\text { E pred severe } \\
5 \\
\end{array}$ \\
\hline Moderate (M) & $\begin{array}{c}\text { mEMB } \\
\text { B pred moderate } \\
7\end{array}$ & $\begin{array}{c}\text { MEMB } \\
\text { Mixed moderate } \\
8\end{array}$ & $\begin{array}{c}\text { SEMB } \\
\text { E pred severe } \\
2 \\
\end{array}$ \\
\hline Severe (S) & $\begin{array}{c}\text { mESB } \\
\text { B pred severe } \\
0\end{array}$ & $\begin{array}{c}\text { MESB } \\
\text { B pred severe } \\
0\end{array}$ & $\begin{array}{c}\text { SESB } \\
\text { Mixed severe } \\
0\end{array}$ \\
\hline
\end{tabular}

quantification (Figure 1). LAA is believed to be the CT correlate of loss of lung recoil representing emphysema at macroscopic or microscopic level. We observed that centrilobular emphysema was the most common subtype, seen either alone $(N=19,36.5 \%)$ or in combination with paraseptal emphysema $(N=18,34.6 \%)$. This type of emphysema is most commonly associated with cigarette smoking. In our study all the 37 patients showing centrilobular emphysema were smokers. Among the rest of patients, $17.3 \%(N=9)$ showed panacinar pattern, while $11.5 \%(N=6)$ showed isolated paraseptal emphysema. The severity of emphysema was stratified on the percentage of lung LAA and categorised into mild (0-5\%), moderate $(5-30 \%)$, and severe (>30\%). The majority of patients showed moderate severity disease $(N=24,46.2 \%)$ followed by mild severity $(N=16,30.8 \%)$. Severe cases were relatively uncommon in our study $(N=7,13.5 \%)$. A statistically significant association was observed between spirometry (FEV1/FVC) and the CT correlate of emphysema (\%LAA), suggesting that patients with more severe obstruction had a greater degree of emphysematous changes. Similar observations were made also for the bronchitis-predominant group, but the correlation was not statistically significant.

CT-based quantitative assessment of airway-predominant disease relies on indices like BWT and PWA. It is believed that these indices reflect the remodelling of small airways, which is typified by increased wall thickness and narrowing of the luminal diameter. Histological exam-
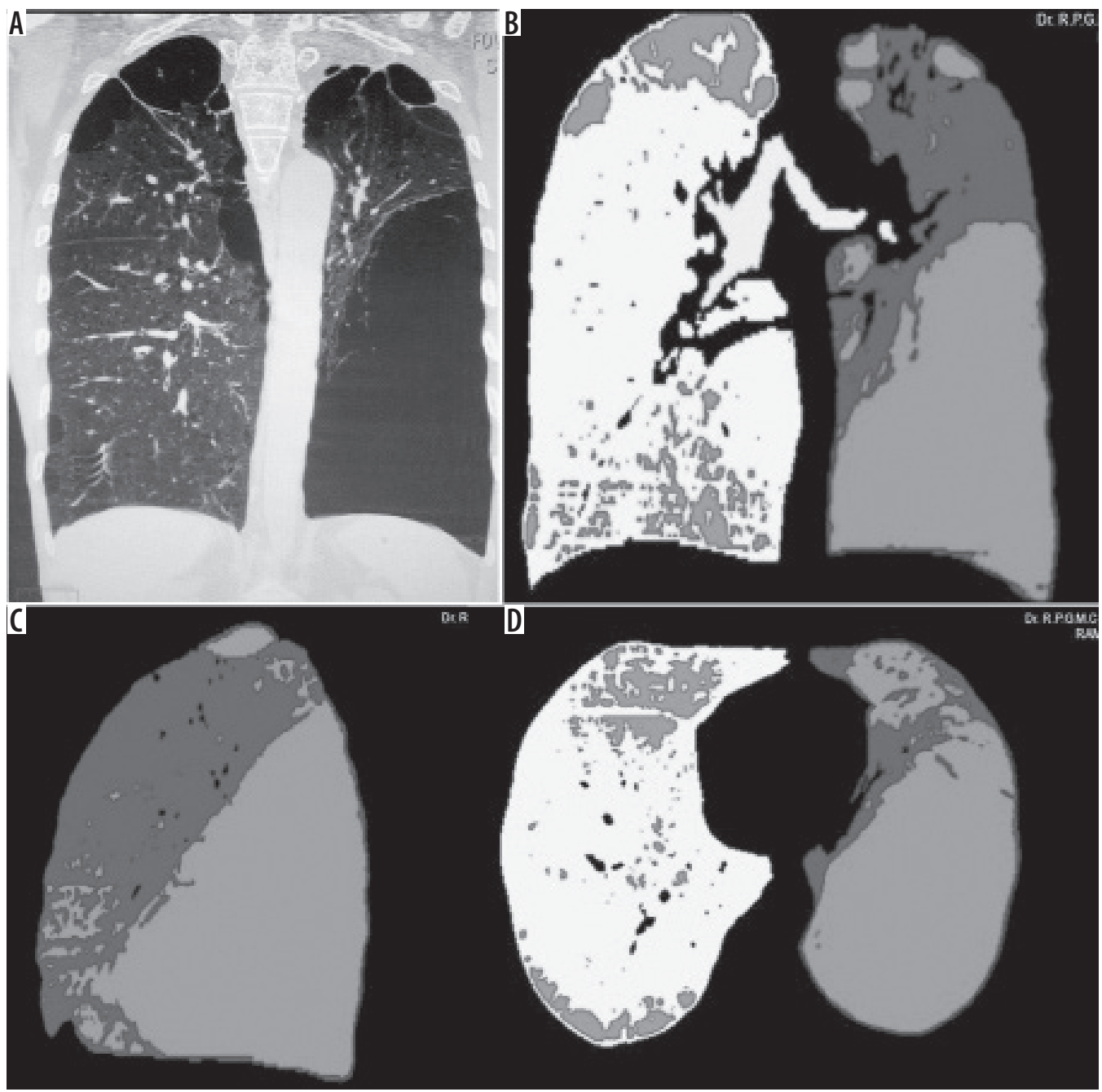

Figure 1. Multi detector computed tomography (MDCT) coronal MPR image (A), coronal density mask images in coronal (B), sagittal (C), and axial planes (D) show a predominantly left-sided emphysematous disease with bullae formation 
ination suggests that the morphological changes in peripheral fifth- to sixth-generation airways have a stronger correlation with the lung function test $[12,13]$. However, since the small airways are not visible on helical CT, it is practical to measure instead the more amenable larger airways. The underlying pathophysiological processes causing airway disease affects both small and large airways, and so measurement of CT indices in large bronchi can reflect the small airway changes too. Previous studies have also observed that cartilaginous airway wall thickening correlated with small airway changes and hence can be used as an alternative for airway inflammatory quantifi- cation $[2,14]$. Because the majority of airways run tangentially to the axial planes and the measurement of airway indices can be attempted only on the perpendicularly sectioned airways with circular configuration, various techniques have been devised to avoid this limitation. These include correcting the error by measuring the angle of deviation [15] or fitting an ellipse in the lumen [16]. Automatic 3D segmentation of bronchial tree airway reconstruction is another popular tool.

We used multiplanar reconstructions from the volumetric data set to align the tangential third and fourth generation bronchi to a perpendicular plane and then

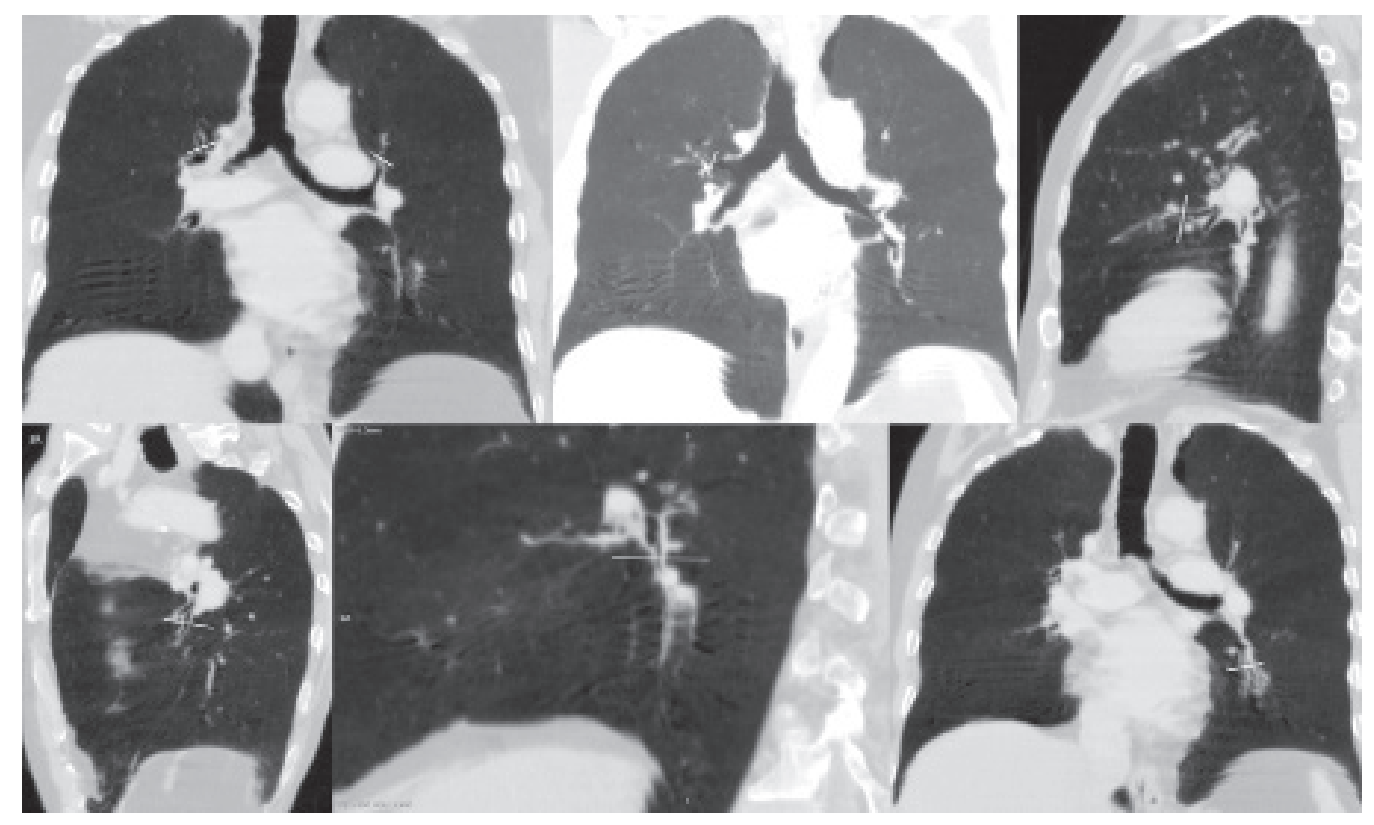

Figure 2. Representative MinIP images showing the various level of sectioning of segmental and subsegmental bronchi (Boyden $3^{\text {rd }}$ and $4^{\text {th }}$ generation) for CT morphometric measurements. At least six bronchi (three in each lung) having circular configuration, diameter more than $2 \mathrm{~mm}$, and vessel contact of $<50 \%$ of circumference were chosen

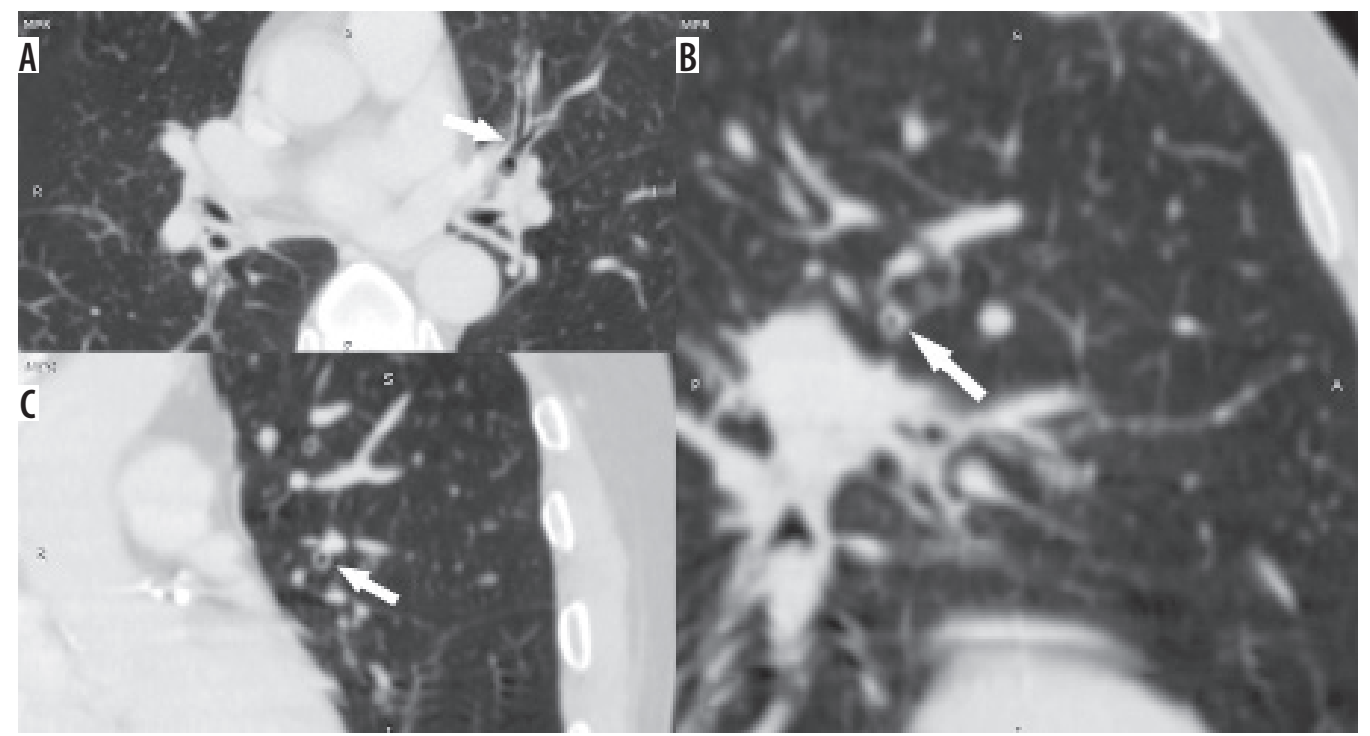

Figure 3. Axial (A), coronal (B), and sagittal (C) images in lung window showing the circumferential wall thickening with luminal narrowing (arrows) in a patient with bronchitis predominant disease 
took the airway measurements in six different levels in each patient (Figures 2, 3). The overall mean PWA was $81.39 \pm 8.53 \%$ and mean BWT was $1.89 \pm 0.42 \mathrm{~mm}$. Mean bronchial wall thickness in the emphysema-predominant subgroup was $1.79 \pm 0.23 \mathrm{~mm}$ and in airway predominant group it was $1.94 \pm 0.28 \mathrm{~mm}$. The difference between the two groups was statistically significant with a $p$ value of 0.005 . Expectedly, the mean PWA in the airway-predominant phenotype $(80.50 \pm 9.33)$ was also greater than in the emphysema-predominant subtype $(77.84 \pm 3.88)$; however, this difference was not statistically significant. These results indicate that wall thickening is reflective of airway remodelling and is more pronounced in the airway-predominant subgroup. This is also supported by previous studies done to calculate bronchial wall thickening in larger as well as distal airways $[17,18]$. Subjects with emphysema-predominant disease have relatively less severe wall thickness and wall area in comparison to the airway-predominant group, presumably because the nature of pathophysiological changes in smokers preferentially occur at a parenchymal level in emphysema as opposed to airways in the airway (bronchitis) subgroup [19].

The mechanisms underlying the airflow limitation in COPD are different in the bronchitis and emphysema subgroups, but a substantial overlap occurs. In the former, airflow limitation is mainly because of the intrinsic changes in bronchial wall resulting in luminal narrowing, while in the latter it is because of alteration in the elastic recoil of lung and radial tractional forces on the bronchial wall. It is difficult to quantify the relative contributions of the two mechanisms because patients often show both of these changes to varying degrees. Using the obtained CT based parameters (\%LAA and bronchial wall thickness), we subcategorised our patients into emphysema predominant, bronchitis predominant, and mixed phenotype, de- pending on the severity of their observed findings (mild, moderate, or severe). Patients showing both the components in equal measure were include in the mixed category. We believe that this concept can be utilised in future studies because it conveys the overlapping and continual nature of findings rather than the past concept of categorising patients into two distinct groups of bronchitis or emphysema alone, which ignores the overlapping minor component on either side.

Our study has some limitations, one of which is the lack of confirmation on histopathological examination. Therefore, the conclusions of our study are observational in nature. Another limitation could be the anatomical heterogeneity, which is believed to occur during the remodelling process in patients with airway disease, resulting in non-uniform changes in the airways.

\section{Conclusions}

Multi detector computed tomography can play a pivotal role in complete evaluation of COPD through assessment of various morphometric and software-generated parameters. Measurement of airway parameters like BWT and PWA should routinely be calculated in all emphysema patients in addition to the LAA percentage, in order to assess the coexisting airway component, grade the disease severity, and accurately categorise COPD into its various phenotypes like emphysema predominant, airway predominant, and mixed type. This simple change of protocol can serve as a valuable tool in initiating appropriate and individualised management strategies for these patients.

\section{Conflict of interest}

The authors report no conflict of interest.

\section{References}

1. Milne S, King GG. Advanced imaging in COPD: insights into pulmonary pathophysiology. J Thorac Dis 2014; 6: 1570-1585.

2. Brillet PY, Fetita CI. Investigation of airways using MDCT for visual and quantitative assessment in COPD patients. Int J Chron Obstruct Pulmon Dis 2008; 3: 97-107.

3. Yamashiro T, Moriya H, Tsubakimoto M, et al. Continuous quantitative measurement of the proximal airway dimensions and lung density on four-dimensional dynamic-ventilation CT in smokers. Int J Chron Obstruct Pulmon Dis 2016; 11: 755-764.

4. Orlandi I, Moroni C, Camiciottoli G, et al. Chronic obstructive pulmonary disease: thin section CT measurement of airway wall thickness and lung attenuation. Radiology 2005; 234: 604-610.

5. Jindal SK, Malik SK. Smoking index - a measure to quantify cumulative smoking exposure. Lung India 1988; 6: 195-196.

6. GuerraS, Sherrill DL, Bobadilla A, et al. The relation of body mass index to asthma, chronic bronchitis and emphysema. Chest 2002; 122: $1256-1263$.
7. Stratelis G, Fransson SG, Schmekel B, et al. High prevalence of emphysema and its association with BMI: a study of smokers with normal spirometry. Scand J Prim Health Care 2008; 26: 241-247.

8. Jain NK, Thakkar MS, Jain N, et al. Chronic obstructive pulmonary disease: does gender really matter? Lung India 2011; 28: 258-262.

9. Boschetto P, Miniati M, Miotto D, et al. Predominant emphysema phenotype in chronic obstructive pulmonary. Eur Respir J, 2003; 21:450-4.

10. Gupta PP, Yadav R, Verma M, et al. High-resolution computed tomography features in patients with chronic obstructive pulmonary disease. Singapore Med J 2009; 50: 193.

11. Matsuoka S, Yamashiro T, Washko GR, et al. Quantitative CT assessment of chronic obstructive pulmonary disease. Radiographics 2010; 30: 55-66.

12. Yanai M, Sekizawa K, Ohrui T, et al. Site of airway obstruction in pulmonary disease: direct measurement of intrabronchial pressure. J Appl Physiol 1992; 72: 1016-1023. 
13. Gurney JW, Jones KK, Robbins RA, et al. Regional distribution of emphysema: correlation of high-resolution CT with pulmonary function tests in unselected smokers. Radiology 1992; 183: 457-463.

14. Tiddens HA, Pare PD, Hogg JC, et al. Cartilaginous airway dimensions and airflow obstruction in human lungs. Am J Respir Crit CareMed 1995; 152: 260-266.

15. King GG, Muller NL, Whittall KP, et al. An analysis algorithm for measuring airway lumen and wall areas from high-resolution computed tomographic data. Am J Respir Crit Care Med 2000; 161: 574-580.

16. Saba OI, Hoffman EA, Reinhardt JM. Maximizing quantitative accuracy of lung airway lumen and wall measures obtained from X-ray CT imaging. J Appl Physiol 2003; 95: 1063-1075.
17. Laura Pini, Valentina Pinelli, Denise Modina, et al. Central airways remodeling in COPD patients, Int J Chron Obstruct Pulmon Dis 2014; 9: 927-933.

18. Hasegawa M, Nasuhara Y, Onodera $Y$, et al. Airflow limitation and airway dimensions in chronic obstructive pulmonary disease. Am J Respir Crit Care Med 2006, 173: 1309-1315.

19. Patel B, Make B, Coxson HO, et al. Airway and parenchymal disease in chronic obstructive pulmonary disease are distinct phenotypes. Proc Am Thorac Soc 2006; 3: 533. 